\title{
Вычисление вертикального градиента силы тяжести по измерениям двухсферного сверхпроводящего гравиметра
}

\author{
Ю. В. Антонов ${ }^{\text {冈 }}$ \\ Воронежский государственный университет \\ Университетская пл.,1, 394018, Воронеж Российская Федерация
}

\begin{abstract}
Аннотация
Введение: Вертикальный градиент силы тяжести в любой точке Земли определяется изменением силы тяжести по вертикали и играет важную роль при изучении Земли и околоземного пространства. К сожалению, вариометра

для измерения вертикального градиента до сих пор не создано. Сейчас измерения ведутся с помощью гравиметров, расположенных на разных высотах.

Методика: Рассмотрено вычисление значений вертикального градиента на основе измерений силы тяжести двухшаровым сверхпроводящим гравиметром по данным измерений на обсерватории ВFО (Шварцвальд, Германия). Обсуждение результатов: В настоящие момент созданы сверхпроводящие гравиметры с двумя датчиками на разных высотах, которые позволяют измерять градиент с высокой точностью. Синхронность датчиков легко согласуется по собственным измерениям. Градиент зависит от наличия метеорных потоков, атмосферных фронтов и других космических и атмосферных явлений.

Bblвoдbl: В результате исследований можно утверждать, что пульсации вертикального градиента существуют наравне с пульсациями силы тяжести и в тех временных рамках, в которых существуют пульсации силы тяжести. Следовательно, источником для пульсаций вертикального градиента являются метеорные потоки и метеорологические факторы (тайфуны, ураганы и т.д.).

Ключевые слова: вертикальный градиент силы тяжести лунно-солнечные вариации силы тяжести; неприливные вариации силы тяжести; корональные выбросы масс; метеорные потоки; пульсации. Для циттирования: Антонов Ю. В. Вычисление вертикального градиента силы тяжести по измерениям двухсферного сверхпроводящего гравиметра // Вестник Воронежского государственного университета. Серия: Геология. 2020. №3. С. 55-63. DOI: https://doi.org/10.17308/geology.2020.3/3009
\end{abstract}

\section{Введение}

Вертикальный градиент в любой точке Земли определяется изменением силы тяжести по вертикали. Чтобы определить вертикальный градиент инструментально, достаточно на поверхности Земли измерить на двух высотах значение силы тяжести, взять разность этих значений и разделить на разность высот. Однако до сих пор не удалось создать портативного прибора для полевых работ, позволяющего измерять эту производную с нужной точностью. Но в настоящее время появились стационарные приборы, которые позволяют с высокой точностью вычислять изменение вертикального градиента во времени. Данные этих измерений находятся в открытом доступе [1].

Первые измерения градиента [2, 3] проводились взвешиванием масс на разных высотах. Все эти опыты, не вполне дававшие желательную точность до нескольких этвеш, были произведены с аппаратурой совершенно не экспедиционного типа. Для портативности и компактности инструмента желательно, чтобы разность высот была невелика (не больше 0.5 м).

Не останавливаясь детально на всех возможных

Контент доступен под лицензией Creative Commons Attribution 4.0 License.

\footnotetext{
Антонов Юрий Васильевич, e-mail: yuriyantonov@yandex.ru
} 
принципах создания вертикальных градиентометров, используемых для теоретических разработок, отметим, что наиболее реальной из них представлялась схема сдвоенных гравиметров [4]. Вместе с тем создание такой схемы существенно осложняет необходимость соблюдения условия полной идентичности параметров спаренных гравиметров во времени, что довольно трудно осуществить, используя пружинные или кварцевые системы, параметры которых изменяются по индивидуальным законам. Иными словами, при использовании для указанных целей гравитационно-чувствительных систем, применяемых в настоящее время для наземных съемок высокой точности, решение поставленной задачи технологически затруднительно

\section{Практические измерения вертикального градиента силы тяжести}

Единственным путем измерения вертикального градиента оставался путь измерения силы тяжести на двух высотах с очень высокой точностью, а высокоточных гравиметров до середины прошлого века не существовало. Оставался единственный способ повышения точности измерений градиента: увеличение разности высот. Поэтому впервые измерения вертикального градиента были проведены 3. Хаммером на высотных зданиях Нью-Йорка, Вашингтона и Питсбурга. Как ни странно, но во всех этих пунктах измеренные значения градиента практически совпали с теоретическими. Максимальное расхождение составило 38 этвеш в Вашингтоне. Видимо, это обстоятельство и предопределило в дальнейшем некоторое охлаждение к массовым измерениям вертикального градиента. Второй заслуживающей внимания попыткой измерения градиента являются измерения Б. К. Балавадзе, который измерил градиент на территории Грузии на 45 триангуляционных вышках [5]. Разность высот составила более 10 м. Эти наблюдения показали, что измеренные значения градиентов могут отличаться от теоретического значения до 300 этвеш. Авторы указанных исследований подобные отклонения объяснили влиянием приповерхностных масс. Позже такие работы в Советском Союзе практически были прекращены. Из появившихся в печати публикаций, пожалуй, следует отметить сообщение о вычислении вертикального градиента на основе измерений силы тяжести в скважинах. Вертикальный аномальный градиент оказался очень большим и не мог быть объяснен геологическим строением изучаемой площади.

Из последующих работ важными являются измерения, проведенные в канадской провинции Альберта и на плоскогорье американского штата Техас, где зафиксированы значительные отклонения градиентов от стандартного значения (до 200 этвеш). Указанные отклонения объясняются наличием приповерхностных неоднородностей с избыточной плотностью до 0.5

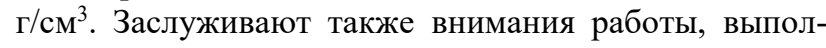
ненные в Польше 3. Файклевичем [6]. Проведено несколько тысяч измерений преимущественно для реше- ния инженерно-геологических задач на небольших площадях»

На юго-западе Британской Колумбии (Канада) выполнена съемка вертикального градиента путем измерений силы тяжести на вышке (0.876 м) [7]. Исследователи выполнили региональный профиль длиной 430 км и провели детальные работы на угольных месторождениях. Хорошая геологическая и физическая изученность этих месторождений определила их в качестве эталона для изучения информативности метода.

На региональном профиле в южной части Британской Колумбии значения вертикального градиента оказались на 300-500 этвеш ниже стандартного значения. Исследователи пришли к выводу, что значение теоретического вертикального градиента для данного района слишком велико, а значение $\mathrm{V}_{\mathrm{zz}}$ в пределах 2600 2800 этвеш будет соответствовать действительности. Эти результаты показывают важность проведения измерений градиента на больших территориях с целью получения действительных значений $\mathrm{V}_{\mathrm{zz}}$ необходимых для редуцирования аномалий силы тяжести при постановке детальных гравиметрических работ.

К подобным выводам в региональном аспекте пришёл автор данной статьи, анализируя материалы гравиметрических съемок в горных районах Таджикистана [8-10]. Между рельефом и аномалиями силы тяжести в редукции Буге при стандартном значении вертикального градиента существует тесная корреляционная связь, которая иногда превышает I мГал на 100 м. Причем наблюдается четкая закономерность: в высокогорных районах эта связь положительная, а в относительно пониженных - отрицательная. В статье специально приведены примеры, отражающие противоположные связи дневного рельефа с аномалиями силы тяжести в редукции Буге.

Необходимо выделить одну из особенностей истолкования геологических результатов Балавадзе Б. К. Он отметил большое несовпадение измеренных аномалий градиента с геологическим строением, но объяснил это несовпадение недостаточной изученностью плотности геологического разреза. Файклевич, проводивший исследования над штольнями, тоже отметил слишком большие амплитуды аномалий градиента. Он объяснил их разуплотнением горных пород над штольней за счет тектонических напряжений, приводящих к образованию трещиноватости после проходки штольни.

Впоследствии под руководством автора данной статьи были проведены обширные работы по измерению вертикального градиента. В частности, выполнены измерения на региональных профилях: Пяндж (граница между Таджикистаном и Афганистаном) - Голодная степь (Узбекистан), профили через Памир и Большой Кавказский хребет, профиль Алушта (Крым) - Шацк (Рязанская губерния), съемка масштаба 1:200 000 на территории Южного Тянь-Шаня (Таджикистан) и съемка на всей территории Воронежского кристаллического массива. Помимо этого, были выполнены многочисленные работы на рудных и нефтяных объектах России (Архангельск, Волгоград, Воронеж, Самара, Якутия). 
Работы проводились с помощью пружинного гравиметра ГНУ-КА (конструкция Веселова К. Е.) на вышке $(0.7$ м). Чтобы получить измерения с погрешностью в 10-15 этвеш необходимо было затрачивать около часа времени на одном пункте наблюдения. Работы требовали больших затрат времени. Позже стали известны причины больших изменений градиента: пульсации за счет метеорных потоков, прохождения атмосферных фронтов, изменения температуры и давления в атмосфере. Но эффективность этих работ, несмотря на большие затраты, оказалась не только в прикладном, но и в теоретическом аспектов очень высокой [8-10]. Поэтому создание приборов для измерения вертикального градиента на сегодня остается актуальным [11].

\section{Методика обработки и обсуждение результатов}

Для примера рассмотрим вначале вычисление значений вертикального градиента на основе измерений силы тяжести двухшаровым сверхпроводящим гравиметром. В настоящее время в открытом доступе имеются подобные измерения по обсерватории ВFО (Шварцвальд, Германия) $[1,4]$.

Исходные данные по измерениям гравиметров в [1] представлены наблюденными значениями размерностью в 7 порядков. Датчики гравиметра (сферы) расположены по высоте друг от друга на 20 см. Цены делений датчиков разнятся в два раза. Разницу абсолютно ровно в два раза подобрать очень сложно. Поэтому соотношение цен деления сфер можно определить следующим путем. Изменение вертикального градиента во времени можно представить в виде:

$$
\mathrm{V}_{\mathrm{ZZ}}(\mathrm{t})=\mathrm{V}_{\mathrm{H}}(\mathrm{t})-\mathrm{K} * \mathrm{~V}_{\mathrm{B}}(\mathrm{t})
$$

где $\mathrm{V}_{z z}(\mathrm{t})$ - вычисленные значения вертикального градиента силы тяжести; $\mathrm{V}_{\mathrm{H}}(\mathrm{t})$ - измеренные значения силы тяжести на нижней сфере; $\mathrm{V}_{\mathrm{B}}(\mathrm{t})$ - измеренные значения силы тяжести на верхней сфере.

Меняя переходной коэффициент К вблизи значения 2, строим графики $\mathrm{V}_{\mathrm{zz}}$. Графики (рис. 1) при изменении коэффициента К достаточно сильно меняют свою морфологию, и оптимальное значение легко определяется. Примерно таким же образом определяется цена деления датчиков. Наблюденные значения силы тяжести практически полностью определяются лунно-солнечными вариациями. Значения притяжения Луны и Солнца для территории, где находится обсерватория, колеблются в пределах \pm 0.15 мГал, а неприливные вариации редко превышают \pm 0.05 мГал. Неприливные вариации с большой амплитудой во времени непродолжительны. Лунно-солнечные вариации могут быть вычислены теоретически. Поэтому для определения цены деления, не вдаваясь в сложные вычисления, достаточно взять отношение теоретических и наблюденных значений в экстремальных точках, где нет заметных неприливных вариаций. К тому же, определив цену деления для обеих сфер, можно проконтролировать коэффициент К. Чтобы не оспаривать точность определения цены деления датчиков на всех графиках значения Vzz даются в делениях прибора, что абсолютно не влияет на выводы.
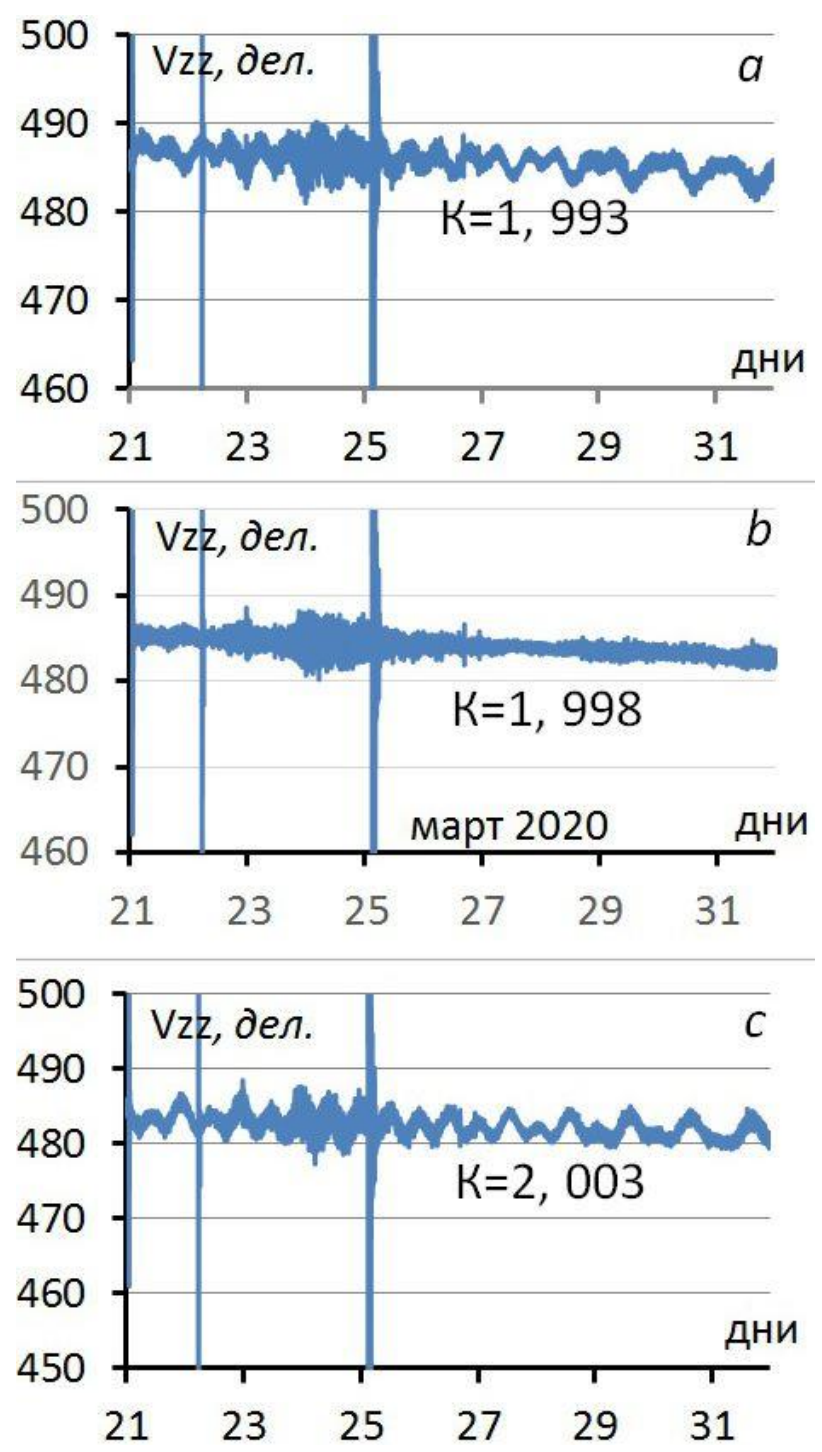

Рис. 1. Подбор коэффициента К при вычислении вертикального градиента силы тяжести: $a$ - кривая градиента при значении $\mathrm{K}=1.993 ; b-$ то же самое при $\mathrm{K}=1.998 ; c-$ то же самое при $\mathrm{K}=2.003$.

[Fig. 1. Selection of the $\mathrm{K}$ coefficient for calculating the vertical gravity gradient: $a$ - gradient curve at $\mathrm{K}=1.993 ; b$ - the same at $\mathrm{K}$ $=1.998 ; c$ - the same at $\mathrm{K}=2.003$.]

На рис. 2 приведены графики изменения вертикального градиента силы тяжести во времени с шагом 1 мин. В целом по делениям прибора с учетом цены деления гравиметра амплитуда градиента колеблется в интервале 3000 этвеш. Но здесь имеется особенность, что значения градиента убывают во времени. Судя по скорости убывания градиента, то в течение одного человеческого поколения сила тяжести исчезнет. Здесь мы имеем дело с застарелой болезнью - элементарным смещением нуль-пункта прибора, хотя здесь используется сверхпроводимость.

А теперь рассмотрим, как ведет себя вертикальный градиент при наличии пульсаций силы тяжести. Вначале рассмотрим поведение градиента при наличии небольшой, без сторонних помех пульсации (рис. 3). 


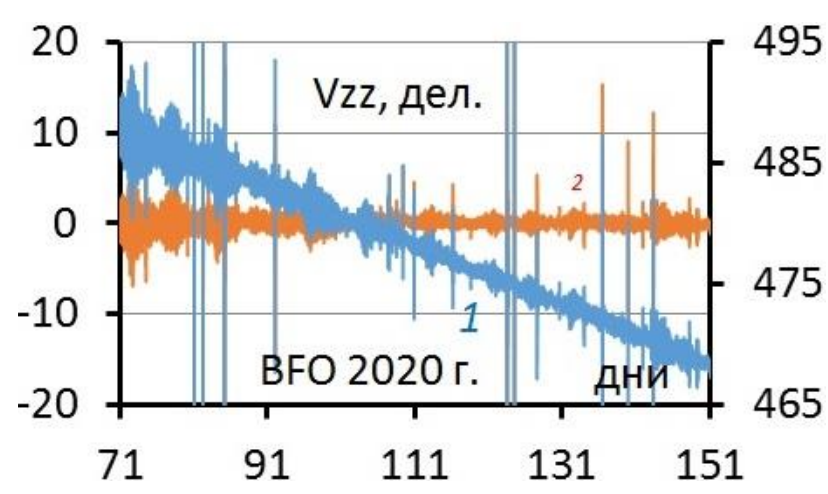

Рис. 2. Характер изменения вертикального градиента силы тяжести во времени 1 - наблюденные значения градиента; 2 - значения градиента после исключения нуль-пункта.

[Fig. 2. the Nature of the change in the vertical gradient of gravity over time 1 - the observed values of the gradient; 2 -the values of the gradient after the elimination of the zero point.]

В начале осени обычно бывает так называемый метеорный поток Сентябрьские Эпсилон Персеиды. Открытый несколько лет назад новый метеорный поток действует по разным оценкам с начала и практически до самого конца сентября. В отличие от августовских Персеид зенитное число на порядок меньше, хотя Сентябрьские эпсилон Персеиды (SPE) - поток не ординарный и это подтверждается двумя неожиданными резкими скачками активности в 2008 и 2013 гг. до 50 вспышек в час. В среднем, почасовая (ZHR) активность Сентябрьских Эпсилон Персеид оценивается на уровне 5-6 метеоров в час. Сентябрьские Эпсилон Персеиды выделяются хорошей яркостью и обладают высокой по метеорным меркам скоростью - около 65 км/сек. Ранее отмечалось [11-13], что амплитуда пульсаций силы тяжести характеризуется не зенитным числом, а общей плотностью метеорного потока. Зенитное число характеризует наличие крупных частиц в потоке. То же самое можно сказать о сентябрьских Персеидах. Максимум вспышек приходится на 9-10 сентября, а максимум амплитуды пульсации силы тяжести приходится на 16 число месяца (рис. 3, $a$ ).

Рассмотрим поведение неприливной вариации силы тяжести (кривая 2 на рис. 3, a) Амплитуда изменения вариаций силы тяжести составляет около 0.05 мГал. Кривая 2 осложнена колебаниями около \pm 0.01 мГал. Колебания силы тяжести, вероятно, связаны с колебаниями атмосферы. Отметим, что неприливная вариация осложнена (рис. $3, a$ ) длиннопериодной аномалией. Природа длиннопериодной помехи легко объяснима. Обратимся к рис. $3, b$, на котором приведены графики наблюденных значений атмосферного давления и дневной температуры [13]. Изменение давления (дискретизация 1 час) хорошо коррелируется (кривая 1 на рис. $3, b$ ) с изменением неприливной пульсации (кривая 2 на рис. $3, a$ ). Увеличение давления ведет к увеличению амплитуды силы тяжести и наоборот.
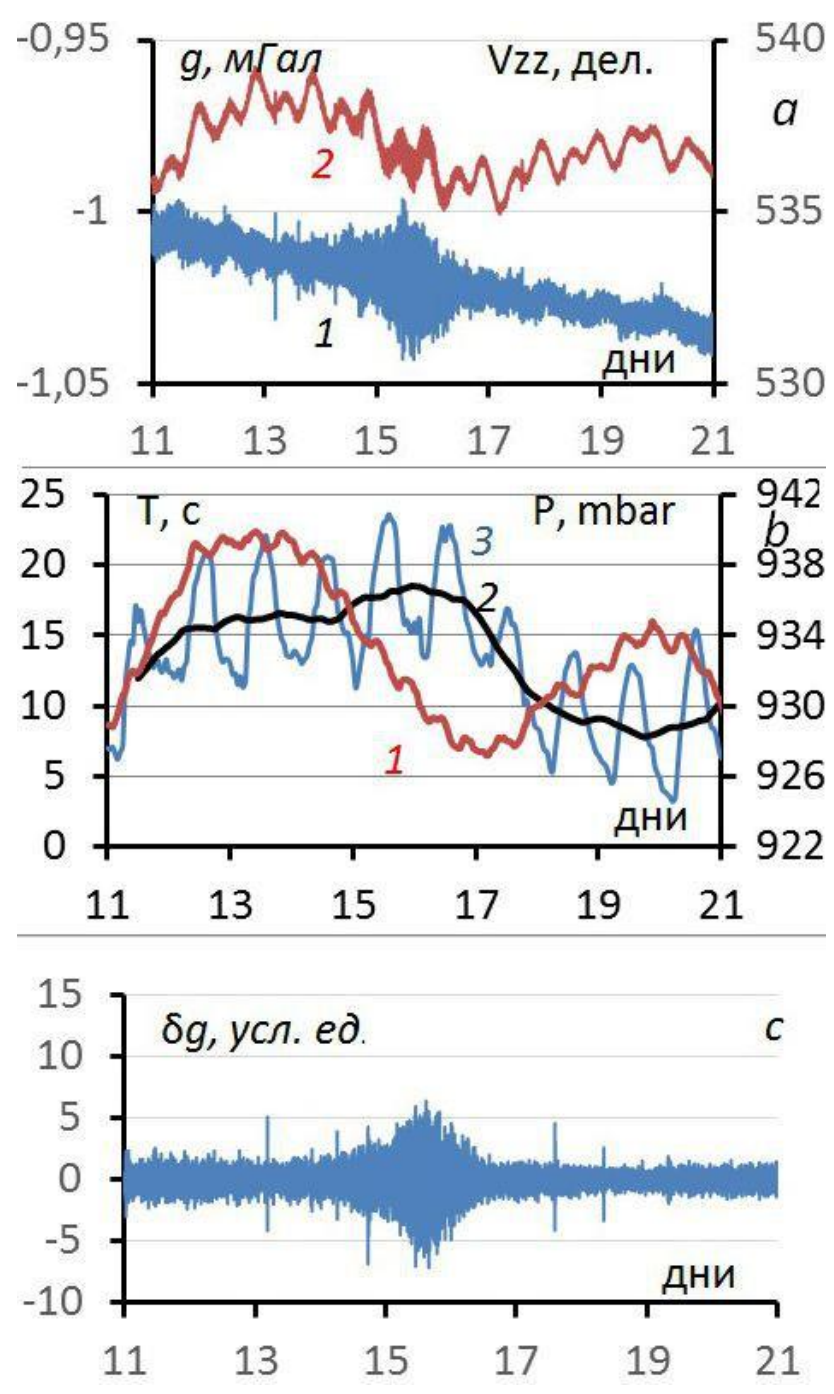

Рис. 3. Сравнение неприливной вариации силы тяжести, вертикального градиента силы тяжести, атмосферного давления и приземной температуры на обсерватории BFO в сентябре 2019 г.: $a$ - неприливная вариация силы тяжести (кривая 1); вертикальный градиент силы тяжести (кривая 2 ); $b$ - распределение атмосферного давления (кривая 1) и приземной температуры (среднесуточной - кривая 2 и текущей - кривая 3); $c$ - остаточная неприливная вариация силы тяжести (пульсация).

[Fig. 3. Comparison of non-tidal gravity variation, vertical gravity gradient, atmospheric pressure, and surface temperature at the BFO Observatory in September 2019: $a$ - non-tidal variation of gravity (curve 1); vertical gradient of gravity (curve 2 ); $b$-distribution of atmospheric pressure (curve 1) and surface temperature (average daily-curve 2 and current-curve 3 ); $c$ - residual non-tidal variation of gravity (ripple).]

С влиянием температуры на гравиметрические измерения дело обстоит несколько иначе [14]. Температура воздуха измеряется на поверхности земли, которая соответствует нескольким десяткам метров воздуха над поверхностью. Температура воздуха меняется в широком диапазоне (кривая 3 на рис. $3, b$ ). Но этого недостаточно, чтобы изменить силу тяжести даже на 
0.001 мГал. Поэтому лучше взять среднесуточную температуру, которая отражает температуру гораздо большей толщи атмосферы (кривая 2 на рис. $3, b$ ). Среднесуточный ход температуры определяется многокилометровой толщей атмосферы [15] Дневная температура изменяется в широком диапазоне, но это не влияет на измеренные значения. Дело в том, что температура измеряется на поверхности Земли и отражает состояние только приземного слоя воздуха. Если взять среднесуточные изменения температуры воздуха, то ее ход находится внешне в противофазе с давлением. В целом же это правило противофазности между среднесуточным ходом температуры и давлением чаще всего соблюдается. Если проанализировать более тщательно эту противофазность, то отмечается сдвиг по фазе. Максимум температурного хода соответствует максимуму градиента изменения давления и наоборот. Что является первичным в связке температура воздуха и давления, то на данном этапе трудно определить.

Чтобы убрать влияние давления атмосферы и температуры, воспользуемся методом осреднения, так как период изменения метеоусловий в несколько раз превышает периодичность пульсаций [11-13]:

$$
\delta g(\mathrm{t})=g \text { изм }(\mathrm{t})-\sum_{-n}^{n} g \text { изм }(\mathrm{t})
$$

где $\delta g(\mathrm{t})$ - исправленная остаточная вариация силь тяжести за давление и температуру; $\sum_{-n}^{n} \Delta g$ изм(t) среднее значение вариации на интервале осреднения (n,n). Интервал осреднения составляет несколько минут (5-10). А на рис. 3, с представлена такая же остаточная вариация силы тяжести, как и н рис. 3, a, вычисленная с помощью метода непосредственно по исходным данным, исключая все промежуточные операции, что намного облегчает обработку измерений [12].

Таким образом, неприливные пульсации вызывают изменение вертикального градиента. Рассмотрим еще один пример, но более сложный, когда присутствует несколько пульсаций силы тяжести (рис. 4). В последней декаде января 2019 г. присутствует несколько неприливных вариаций: 19 января -Альфа Гидриды; 21 января - Эта Кариниды и 30 января Альфа Кариниды и Дельта Велиды. Даты указаны для вспышек метеоров.

Неприливные вариации силы тяжести чаще всего сдвинуты относительно максимумов вспышек. Всем пульсациям соответствуют минимальные значения давления. Вертикальный градиент резко меняется в течение всей недели. Этому способствуют наличие неприливных вариаций (рис. 4, с), непрерывные снегопады, ливни и штормовые ветра (порывы до 17 м/с). Давление меняется часто. Тем не менее, остаточные неприливные пульсации выделяются очень четко (рис. $4, c)$. Эти же пульсации выделяются в обсерватории Арти (рис. 5, a). Но погода в Арти очень морозная, всю декаду практически стоит штиль.

Приведем еще один пример силы тяжести и вертикального градиента в Арти и ВFО (рис. 6). С 22 мая 2020 г. проходит достаточно мощный поток Ариетиды (зенитное число до 60 единиц). На обсерватории ВFО
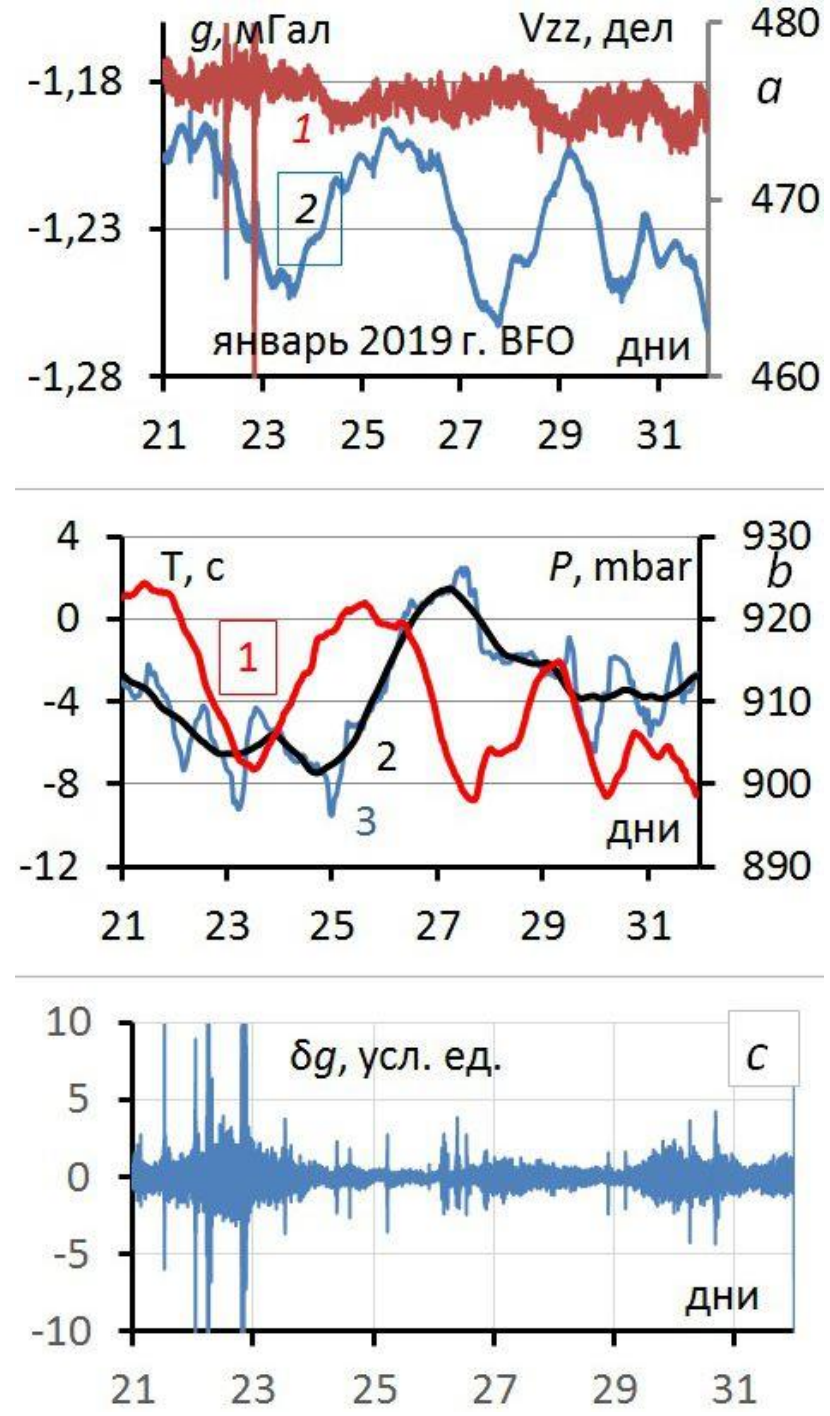

Рис. 4. Сравнение неприливной вариации силы тяжести, вертикального градиента силы тяжести, атмосферного давления и приземной температуры на обсерватории BFO в январе 2019 г. Обозначения те же, что на рис. 3.

[Fig. 4. Comparison of non-tidal gravity variation, vertical gravity gradient, atmospheric pressure, and surface temperature at the BFO Observatory in January 2019.]

поток совпадает с резкой сменой погоды (рис. $6, b)$, поэтому наблюдается довольно мощная пульсация силы тяжести и вертикального градиента. В Арти (рис. $6, d$ ), нет такого перепада в давления и температуры. Но пульсации на обеих обсерваториях все равно наблюдаются.

Наконец, оценим влияние атмосферных фронтов на вертикальный градиент силы тяжести (рис. 7). На рис. 7 приведены данные градиента за 2019 и 2020 гг. Взята специально середина апреля, когда практически не бывает метеорных потоков. В 2019 г. аномалии вертикального градиента нет. А в 2020 г. наблюдается четкая пульсация. Пульсация вызвана атмосферным фронтом. По данным [14] в 2019 осадков (преимущественно снег) было больше, но они проходили при штилевой погоде. Но в 2020 г. в этот же период был лишь один 


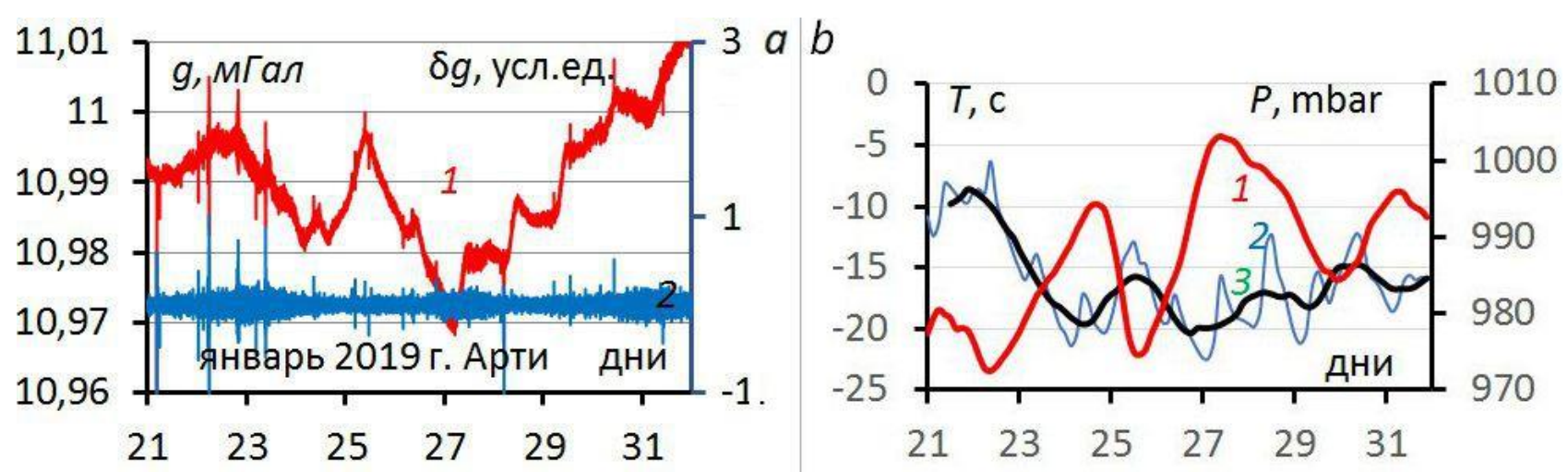

Рис. 5. Сравнение пульсаций силы тяжести в ВFО (а) и Арти (b) в январе 2019 г. Обозначения те же, что на рис. 3.

[Fig. 5. Comparison of gravity ripples in BFO (a) and Arti (b) in January 2019 The designations are the same as in Fig. 3.]
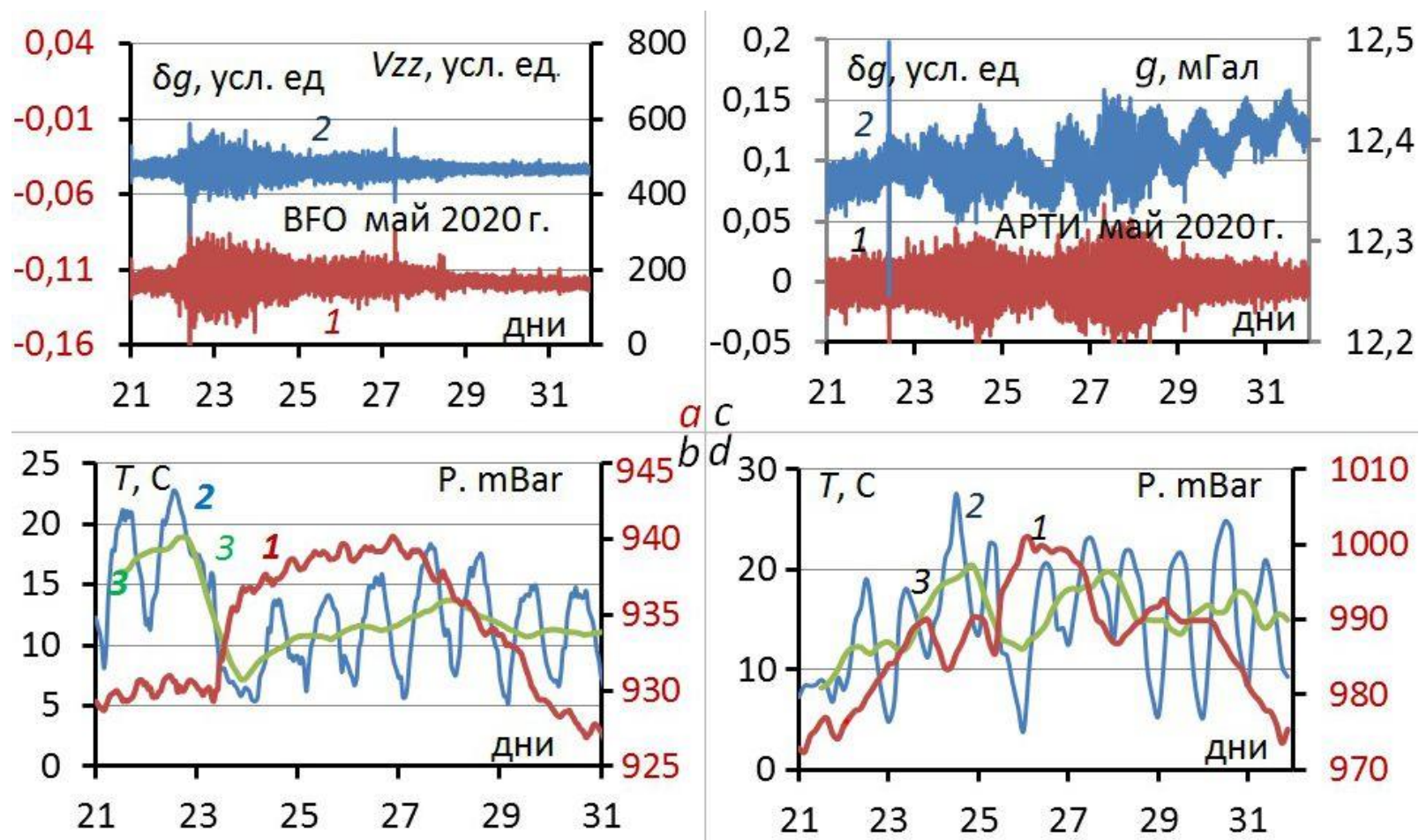

Рис. 6. Сравнение неприливной вариации силы тяжести, вертикального градиента силы тяжести, атмосферного давления и приземной температуры на обсерваториях ВFO и Арти в мае 2020 г.

[Fig. 6. Comparison of non-tidal variation of gravity, vertical gradient of gravity, atmospheric pressure and surface temperature at the BFO and Arti observatories in may 2020.]

дождь (13-14 апреля), но этот дождь сопровождался порывами ветра до 15 м/сек. К этому же времени приурочено падение атмосферного давления (рис $7, d$ ), а также понижение значений неприливной вариации силы тяжести (рис 7, с, кривая 2), Понижение амплитуды вариации обусловлено тем, что атмосфера фронта была с повышенной водностью: избыточная масса влаги находится выше пункта наблюдения и создает отрицательную аномалию.

Пульсации поля, создаваемые атмосферными фронтами, могут возникать на континентах [15], но они по времени непродолжительны. Другое дело появление пульсаций в прибрежных зонах и океанах. Здесь сила природных стихий в разы больше, чем на суше.

\section{Выводы}

В результате можно утверждать, что пульсации вертикального градиента существуют наравне с пульсациями силы тяжести и в тех временных рамках, в которых существуют пульсации силы тяжести. Следовательно, источником для пульсаций вертикального градиента являются метеорные потоки и метеорологические факторы (тайфуны, ураганы и т.д.). Вполне очевидно, что пульсации нужно и можно использовать при проведении полевых работ по измерению вертикального градиента в целях геологоразведки. Можно также использовать для изучения метеорных потоков. В прикладном плане изучение пульсаций внесет определенные коррективы в методику высокоточных измерений силы тяжести. 


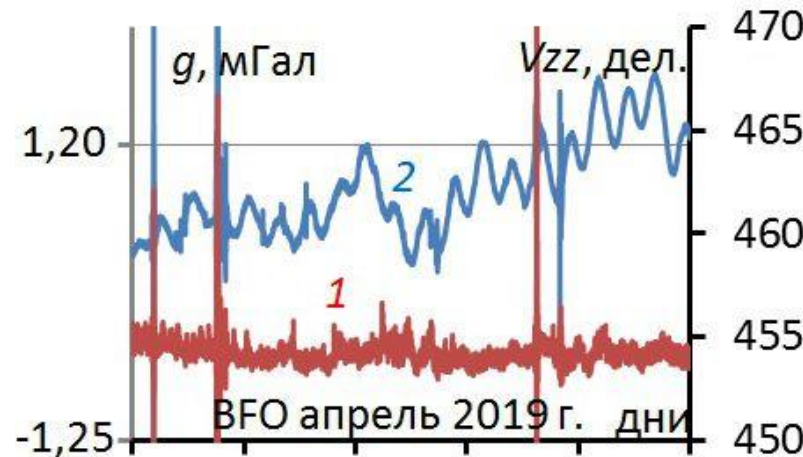

\section{$\begin{array}{llllll}11 & 13 & 15 & 17 & 19 & 21\end{array}$}

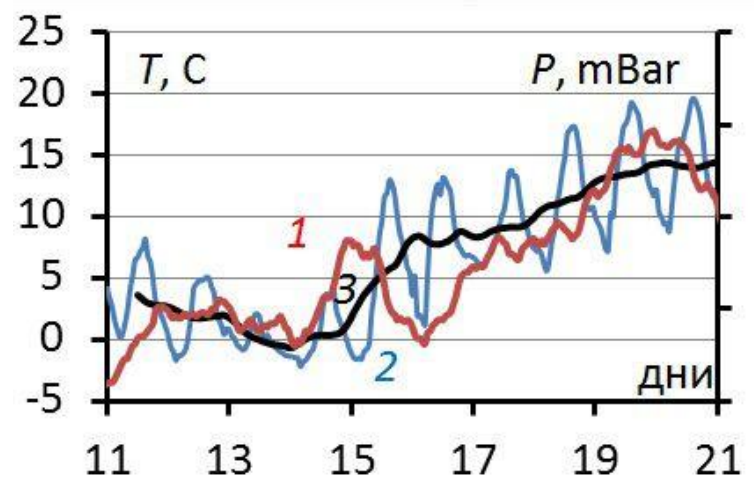

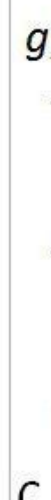

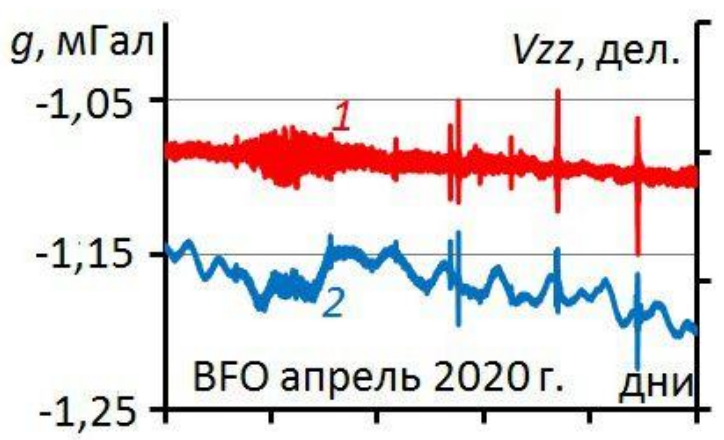

490

480

470

460

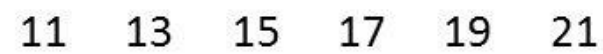

ac

940

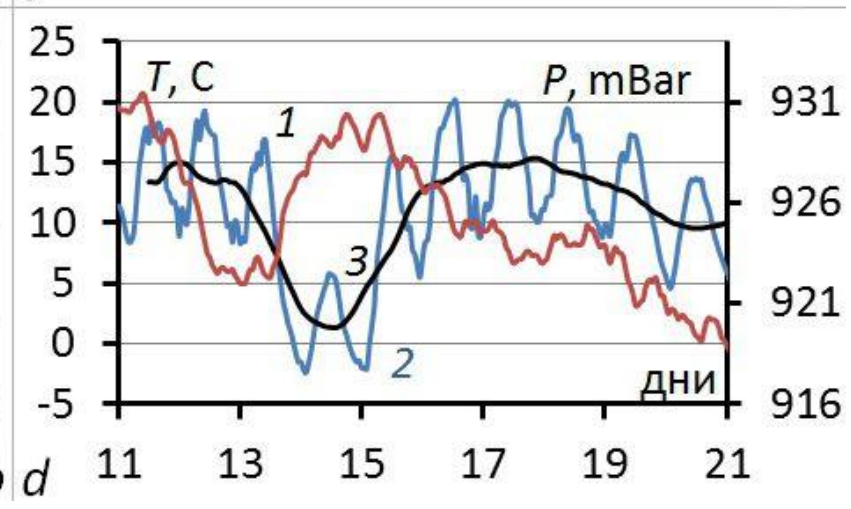

Рис. 7. Сравнение неприливной вариации силы тяжести, вертикального градиента силы тяжести, атмосферного давления и приземной температуры на обсерватории ВFО в апреле 2019 и 2020 гг.

[Fig. 7. Comparison of non-tidal gravity variation, vertical gravity gradient, atmospheric pressure, and surface temperature at the BFO Observatory in April 2019 and 2020.]

Конфликт интересов. Авторы декларируют отсутствие явных и потенциальных конфликтов интересов, связанных с публикацией настоящей статьи.

Благодарности: Автор благодарит за предоставленные гравиметрические материалы Бебнева А. С. (ИГ УрО РАН).

\section{ЛИТЕРАТУРА}

1. Project IDA [Электронный ресурс]. URL:

https://sur.ly/i/ida.ucsd.edu/ (дата обращения: 1.09.2020)

2. Сорокин Л. В. Гравиметрия и гравиметрическая разведка. М., Гостоптехиздат. 1953. $483 \mathrm{c.}$

3. Балабушевич И. А. Высшие производные потециала силы тяжести. Киев, АН УССР. 1963. 267 с.

4. Goodkind J. M The superconducting Gravimeter // Rev. Sci. Instrum. 1999. Vol. 70. No.11. pp. 4131-4152.

5. Балавадзе Б. К. О методике определения вертикального градиента силы тяжести // Изв. АН СССР, сер. геофиз. № 1. 1955. C. $45-55$

6. Fajklevicz S. J. Gravity vertical gradient measurements for the direction of small geologie and anthropogenic forms // Geophysics. 1987. Vol. 41. №5. pp. 1016-1030.
7. Ager C. A., Liard I. O. Vertical Gravity Gradient surveys: Field Reand interpretation in British Columbia. Canada // Geophysics. V.47. №7. 1982. pp. 219-295.

8. Антонов Ю. В., Зубченко Е. А., Савинкин А. Т. Измерение вертикального градиента силы тяжести в Таджикистане // Разведка и охрана недр. 1986. № 9. С. 39-42.

9. Антонов Ю. В. Жаворонкин В. И. Слюсарев С. В. О поверхности изостатического равновесия в верхних слоях земной коры // Геофизика. 2003. № 6. С. 28-38.

10. Антонов Ю. В. Измеренные и вычисленные значения силы тяжести и ее производных// Изв. ВУЗов. Геология и разведка. 2004. № 4. С. 53-57.

11. Антонов Ю. В. Пульсации неприливных вариаций силы тяжести // Изв. ВУЗов. Геология и разведка. 2014. № 5. С. $54-57$

12. Антонов Ю. В., Сизаск И. А. Синхронные пульсации в неприливных вариациях гравитационного и сейсмического полей // Геология и разведка. Изв. ВУЗ. 2015. №5. С. 46-52.

13 Антонов Ю. В. Влияние атмосферного фронта на показания гравиметров и сейсмометров // Геология и разведка. Изв. ВУ3. 2017. №4. С. 66-71.

14. Расписание погоды [Электронный ресурc]. URL: http://rp5.ru (дата обращения: 01.09.2020)

15. Матвеев Л. Т. Физика атмосферы Л.: Гидрометеоиздат, $2000.780 \mathrm{c}$. 
UDC 550.831

DOI: https://doi.org/10.17308/geology.2020.3/3009

ISSN 1609-0691

Received: 25.08 .2020

Accepted: 02.09 .2020

Published online: 30.09 .2020

\title{
Calculation of the vertical gravity gradient using the measurements of a dual-sphere superconducting gravimeter
}

\author{
Yu. V. Antonov ${ }^{凶}$ \\ Voronezh State University \\ 1 Universitetskaya pl., Voronezh 394018, Russian Federation
}

\begin{abstract}
Introduction: The vertical gravity gradient at any point on the Earth is determined by the vertical change in gravity and plays an important role in the study of the Earth and near-Earth space. Unfortunately, a variometer to measure the vertical gradient has not yet been created. Now measurements are made using gravimeters located at different heights.

Methodology: The calculation of the vertical gradient values based on gravity measurements with a dualsphere superconducting gravimeter using the data obtained at the BFO observatory (Schwarzwald, Germany) was considered.

Results and discussion: At the moment, superconducting gravimeters with two sensors at different heights have been created, they make it possible to measure the gradient with high accuracy. The sensors are easily synchronized by their own measurements. The gradient depends on the presence of meteor showers, atmospheric fronts, and other space and atmospheric phenomena.

Conclusions: As a result of the research, it can be said that the pulsations of the vertical gradient exist along with the gravity pulsations and in the same time scale. Consequently, the pulsations of the vertical gradient are caused by meteor showers and meteorological factors (typhoons, hurricanes, etc.).

Keywords: Vertical gravity gradient, lunar-solar variations of gravity, non-tidal variations of gravity, mass coronal ejections, meteor showers, pulsations.

For citation: Antonov Yu. V. Calculation of the vertical gravity gradient using the measurements of a double-sphere superconducting gravimeter. Vestnik Voronezhskogo gosudarstvennogo universiteta. Seriya: Geologiya $=$ Proceedings of Voronezh State University. Series: Geology. 2020. No.3. pp. 55-63. DOI: https://doi.org/10.17308/geology.2020.3/3009
\end{abstract}

Acknowledgments: The author thanks Bebnev A. S. for the gravimetric materials provided (IG UB RAS).

Conflict of interests: The authors declare the absence of obvious and potential conflicts of interest related to the publication of this article

\section{REFERENCES}

1. Project IDA. Available at: https://sur.ly/i/ida.ucsd.edu/ (accessed 1.09.2020)

2. Sorokin L. V. Gravimetria i gravimetricheskaya razvedka [ Gravimetry and gravimetric exploration]. Moscow, Gostoptekhizdat. 1953. 483 p. (In Russ.)

3. Balabushevich I. A. Vysshie proizvodnye potenziala sily tyazhesti [Higher derivatives of the gravity potential]. Kiev, Ukrainian Academy of Sciences. 1963. 267 p. (In Russ.)
4. Goodkind J .M The superconducting Gravimeter. Rev. Sci. Instrum. 1999, vol. 70, no.11, pp. 4131-4152.

5. Balavadze B. K. O metodike opredelenia vertikalnogo gradienta sily tyazhesti [On the method of determining the vertical gradient of gravity]. Izv.AN USSR, ser. Geofiz = Izv. USSR Academy of Sciences, ser. geophysis. 1955, no. 1, pp. 45-55. (In Russ.)

6. Fajklevicz S. J. Gravity vertical gradient measurements for the direction of small geologie and anthropogenic forms. Geophysics. 1987, vol. 41, no. 5, pp. 1016-1030.

\footnotetext{
$\bowtie$ Yuriy V. Antonov, e-mail: yuriyantonov@yandex.ru
} 
7. Ager C. A., Liard I. O. Vertical Gravity Gradient surveys: Field Reand interpretation in British Columbia. Canada. Geophysics. 1982, vol. 47, no. 7, pp. 219-295.

8. Antonov Yu. V., Zubchenko E. A., Savinkin A. T. Izmerenie vertikalnogo gradienta sily tyazhesti v Tajikistane [Measuring the vertical gradient of gravity in Tajikistan]. Razvedka $i$ okhrana $n e d r=$ Exploration and protection of mineral resources, 1986, no. 9, pp. 39-42. (In Russ.)

9. Antonov Yu. V. Zhavoronkin V. I. Slyusarev S. V. O poverkhnosti izostaticheskogo ravnovesia $\mathrm{v}$ verkhnikh sloyakh zemnoy kory [On the surface of isostatic equilibrium in the upper layers of the earth's crust]. Geophysics. 2003, no. 6, pp. 28-38. (In Russ.)

10. Antonov Yu. V. Izmerennye I vychislennye znachenia sily tyazhesti i ee proiiizvodnykh [Measured and calculated values of gravity and its derivatives]. Izv. VUZov. Geologiya $i$ razvedka $=$ Izv. higher educational. Geology and exploration. 2004, no. 4, pp. 53-57. (In Russ.)
11.Antonov Yu. V. Pul'satsii neprilivnykh variatsiy sily tyazhesti [Ripple tidal gravity variations]. Izv. VUZov. Geologiya $i$ razvedka = Izv. higher educational. Geology and exploration., 2014, no. 5, pp. 54-57. (In Russ.)

12. Antonov Yu. V, Sizask I. A. Sinkhronnye pul'satsii v neprilivnykh variatsiyakh gravitatsionnogo i seysmicheskogo poley [Synchronous ripples innon-tidal variations of gravitational and seismicfields]. Izv. VUZov. Geologiya i razvedka $=I z v$. higher educational. Geology and exploration, 2015, no. 5, pp. 46-52. (In Russ.)

13. Antonov Yu. V. Vliyanie atmosfernogo fronta na pokazaniya gravimetrov i seymometrov [Influence of the atmospheric front on the readings of gravimeters and seismometers]. Izv. VUZov. Geologiya i razvedka $=I z v$. higher educational. Geology and exploration, 2017, no. 4, pp. 66-71. (In Russ.)

14. Raspisanie pogody [Weather schedule]. Available at: http://rp5.ru archive (accessed 1 september 2020)

15. Matveev L. T. Phyzika atmosfery [Atmospheric physics]. Saint-Petersburg, Gidrometeoizdat, 2000. 780 p. (In Russ.)
Антонов Юрий Васильевич - д. тех. н., профессор, Воронежский государственный университет, Воронеж, Российская Федерация; E-mail: yuriyantonov@yandex.ru; ORCID http://orcid.org/0000-0002-3323-9697

Автор прочитал и одобрил окончательный вариант рукописи.
Yuriy V. Antonov - Dr. habil. in Techn., Professor, Voronezh State University, Voronezh, Russian Federation;

E-mail: yuriyantonov@yandex.ru;

ORCID http://orcid.org/0000-0002-3323-9697

Author have read and approved the final manuscript. 